\title{
Der asymptomatische Patient mit schwerer Aortenstenose oder Mitralinsuffizienz: wann operieren?
}

\section{The Patient with Asymptomatic Severe Aortic Stenosis or Mitral Regurgitation: When to Operate?}

\author{
Autor \\ Gerrit Kaleschke \\ Institut \\ Department für Kardiologie und Angiologie, Klinik für \\ angeborene (EMAH) und erworbene Herzfehler, Universitäts- \\ klinikum Münster \\ Schlüsselwörter \\ asymptomatische Herzklappenerkrankung, asymptomatische \\ Aortenklappenstenose, asymptomatische Mitralinsuffizienz
}

Key words

asymptomatic heart valve disease, asymptomatic aortic stenosis, asymptomatic mitral regurgitation, watchful waiting

Bibliografie

DOI https://doi.org/10.1055/s-0044-100361

Aktuel Kardiol 2018; 7: 45-49 @ Georg Thieme Verlag KG

Stuttgart · New York | ISSN 2193-5203

Korrespondenzadresse

Dr. Gerrit Kaleschke

Department für Kardiologie und Angiologie, Klinik für

angeborene (EMAH) und erworbene Herzfehler, Universitätsklinikum Münster

Albert-Schweitzer-Campus 1, 48149 Münster

Tel.: 0251/83-46110, Fax: 0251/83-46109

gerrit.kaleschke@ukmuenster.de

\section{ZUSAMMENFASSUNG}

Während symptomatische Herzklappenfehler nicht nur zur Symptomverbesserung, sondern meistens auch aus prognostischen Gründen eine Behandlungsindikation haben, ist die Situation bei noch asymptomatischen Klappenfehlern weniger eindeutig und zum Teil auch umstritten. Die Empfehlungen basieren vor allem auf Prädiktoren für ein schlechteres Outcome im Spontanverlauf oder nach Operation, wie beispiels- weise eine progrediente, unter Umständen irreversible Schädigung von Ventrikelmyokard oder Pulmonalgefäßbett. Auch schwere Vitien können oft lange Zeit asymptomatisch bleiben. Ein Abwarten unter Beobachtung („watchful waiting“) kann dann eine sichere Vorgangsweise sein, wenn regelmäßige Kontrollen, die das rechtzeitige Erkennen von prognostisch ungünstigen Zeichen und in der Folge die zeitgerechte Intervention erlauben, gewährleistet sind. Für die Therapieentscheidung sind dabei das individuelle Eingriffsrisiko selbst, die Möglichkeit einer klappenerhaltenden Operation und die langfristigen potenziellen Komplikationen durch Klappenprothesen wie Endokarditis oder Thrombembolie bzw. Blutungsrisiken unter Antikoagulation zu berücksichtigen. Die aktuellen Leitlinien der ESC/EACTS geben praktische Hinweise und beziehen multimodale diagnostische Strategien in die Empfehlungen ein.

\section{ABSTRACT}

While indication for surgery or intervention in symptomatic heart valve disease is clearly defined in most cases, decision making in asymptomatic patients is often more complicated. The recommendations for treatment are mostly based on predictors for worse outcome in the course of the disease with or without operation (e.g. worsening of ventricular function or progressive enlargement, pulmonary hypertension). Nevertheless, even severe heart valve defects such as asymptomatic aortic stenosis or primary mitral regurgitation might - in absence of predictors for worse outcome - be managed by using a "watchful waiting" strategy with low risk for the patient, but adequate examination intervals have to be assured. Diagnostic strategies have to imply multiple clinical and technical aspects and in some cases different imaging modalities. Treatment options have to be weighted against procedural and long-term risks of heart valve replacement such as endocarditis, bleeding or thromboembolism. 


\section{WAS IST WICHTIG?}

- Bei der Behandlung asymptomatischer Klappenfehler muss der prognostische Benefit das Eingriffsrisiko und mögliche Langzeitkomplikationen nach Operation (Endokarditis, Thrombembolien, Blutung unter Antikoagulation, mangelnde Prothesenhaltbarkeit) aufwiegen. Die Möglichkeit einer klappenerhaltenden OP sowie das Risiko durch Begleiterkrankungen beeinflussen die Indikation und den Behandlungszeitpunkt.

- Unter regelmäßiger Kontrolle („watchful waiting“) können u.U. auch schwere Klappenvitien primär beobachtet werden, sofern das Auftreten ungünstiger Prädiktoren (z. B. Ventrikelvergrößerung, Myokardfunktionsverschlechterung, relevante pulmonale Hypertonie) durch geeignete Untersuchungen ausgeschlossen werden kann.

- Die Kontrollintervalle müssen an die Wahrscheinlichkeit einer Symptomentwicklung und die Dynamik der Klappenerkrankung (z. B. Zunahme der Flussgeschwindigkeit über die Aortenklappe) angepasst werden, um den optimalen OP-Zeitpunkt bestimmen zu können, ohne das Risiko für den Patienten zu erhöhen.

\section{Aortenklappenstenose}

\section{Allgemeines}

Die Aortenklappenstenose (AS) hat eine hohe Prävalenz im fortgeschrittenen Lebensalter von bis zu $10 \%$ bei über 80 -Jährigen und ist der häufigste chirurgisch oder katheterinterventionell behandelte Klappenfehler. Die Progression von der Sklerose bis zur schweren, kalzifizierenden Stenose vollzieht sich meist über Jahre, wobei nicht alle Patienten mit einer reinen Klappensklerose auch wirklich eine fortschreitende Obstruktion erleiden. Entwickelt sich aber eine hämodynamische Auswirkung (Flussbeschleunigung), reduziert sich die Öffnungsfläche weiter (ca. 0,1 cm²/Jahr [1], Anstieg des mittleren Gradienten um ca. $5-8 \mathrm{mmHg} / \mathrm{Jahr}$ ). Unabhängig von der Stenose selbst ist das Vorhandensein einer Aortensklerose assoziiert mit einem erhöhten Risiko für kardiovaskuläre Ereignisse [2].

Die Entscheidung für die Operation einer schweren, asymptomatischen AS besteht eindeutig bei sonst nicht erklärbarer Ventrikel-Funktionseinschränkung oder im Rahmen ohnehin erforderlicher herzchirurgischer Eingriffe (Klappen, Koronarbypass oder Aorta-ascendens-Chirurgie), in solchen Fällen wird auch bei mittelgradiger AS in der Regel mit einem Klappenersatz kombiniert. Die weiteren Szenarien sind bislang weniger eindeutig.

\section{Natürlicher Verlauf und Prognoseparameter}

Selbst schwergradige Stenosen werden von vielen Patienten z.T. jahrelang erstaunlich gut toleriert, etwa die Hälfte der Patienten haben zum Zeitpunkt der Diagnose keine Symptome. Dennoch werden relativ viele Patienten innerhalb absehbarer Zeit symptomatisch, erhalten einen Klappenersatz oder erleiden ein kardiovaskuläres Ereignis (bis zu 85\% nach 5 Jahren [1,3,4]). Verschie- dene Prädiktoren wurden beschrieben, die mit einer rascheren Progredienz der Stenose assoziiert sind: Rauchen, Dyslipidämie, männliches Geschlecht, Diabetes mellitus, arterielle Hypertonie, chronische Niereninsuffizienz, KHK und Verkalkungsgrad der Aortenklappe [5]. Allerdings lassen sich auch aus diesen Parametern keine genauen Prognosen ableiten, in welchem Zeitrahmen die Patienten symptomatisch werden und eine Behandlung benötigen.

Es ist wichtig, die Kontrollintervalle abzustimmen auf die Wahrscheinlichkeit, Symptome zu entwickeln, da diese Entwicklung entscheidend für die Prognose ist. Im Folgenden wird auf verschiedene Modalitäten zur Risikostratifikation eingegangen.

\section{Belastungstests}

Es reicht insbesondere bei älteren Patienten nicht aus, sich auf anamnestische Angaben für eine Belastungseinschränkung zu verlassen, da eine Leistungsminderung oft als schicksalshaft angesehen und die Alltagsaktivitäten entsprechend angepasst werden. Als Ausgangstest ist es deshalb empfehlenswert, sich von der symptomfreien Belastbarkeit zu überzeugen (z. B. beim Treppensteigen). Erschwert wird die Beurteilung hier beispielsweise durch begleitende pulmonale Erkrankungen oder Anämie. Ein nicht unerheblicher Patientenanteil älterer Patienten ist nicht in der Lage, Belastungstests durchzuführen, sodass hier andere Parameter (s.u.) herangezogen werden müssen. Bei körperlich aktiveren Patienten sind objektivierbare Belastungstest (z. B. Spiroergometrie) hilfreich, um Symptome zu demaskieren. Die Wahrscheinlichkeit, dass bei zunächst als asymptomatisch eingestuften Patienten abnorme Belastungstests resultieren, beträgt ca. 50\% (zusammengefasst in [6]). Dazu gehören - neben Dyspnoe - Schwindel oder Angina auch ein systolischer Blutdruckabfall. ST-Streckenveränderungen und komplexe ventrikuläre Arrhythmien erscheinen hingegen wenig spezifisch. Ein pathologischer Stress-Test ist mit einem erhöhten Mortalitätsrisiko assoziiert [7].

\section{Echokardiografische Parameter}

Für eine Reihe von Parametern wurde in Studien eine Assoziation mit der Prognose berichtet. Dazu zählen die transvalvuläre Flussgeschwindigkeit, der Verkalkungsgrad, die Progressionsgeschwindigkeit, die Zunahme des Gradienten unter Belastung, die LVWanddicke, LV-Strain und Pulmonalisdruck. In der Regel sind die dabei vorhergesagten Ereignisse allerdings ganz überwiegend die Entwicklung von Symptomen, die letztlich zu einer Operation führten. Die Parameter sagen also in erster Linie vorher, wie rasch der Patient wahrscheinlich symptomatisch wird. Es bleibt aber unklar, ob eine Intervention bei Erreichen eines der Parameter die Prognose verbessern kann. Eine sehr hohe Flussgeschwindigkeit $\left(V_{\max }>5,5 \mathrm{~m} / \mathrm{s}\right)$ und ausgeprägte Klappenverkalkungen mit raschem Anstieg der Flussgeschwindigkeiten (>0,3 m/s pro Jahr) sprechen für eine so kurze Symptomfreiheit, dass sie in die Leitlinie als Indikation zur Intervention aufgenommen wurden. Grenzwerte für die Myokardhypertrophie sind nach wie vor zu schlecht definiert, und für den Anstieg des mittleren Gradienten unter Belastung um $>20 \mathrm{mmHg}$ konnte in neueren Studien kein zusätzlicher prognostischer Wert zum Ruhegradienten gefunden werden, sodass diese Kriterien wieder gestrichen wurden [8]. Neuere Parameter wie longitudinaler Strain können bei Anzeichen einer 
- Tab. 1 Behandlungsindikationen bei schwerer, asymptomatischer Aortenstenose (Daten aus [8]).
Symptomentwicklung bei Belastungstest mit

Bezug zur Aortenstenose

Aortenklappenersatz im Zusammenhang mit

Bypass- oder Aortenchirurgie

Belastungstest mit Blutdruckabfall unter das

Ausgangsniveau

bei Fehlen der oben angeführten Kriterien und

niedrigem Operationsrisiko:

sehr schwere AS $\left(V_{\max }>5,5 \mathrm{~m} / \mathrm{s}\right)$

schwere Verkalkung und rasche hämodynamische

Progression ( $V_{\max }$-Zunahme $\geq 0,3 \mathrm{~m} / \mathrm{s}$ pro Jahr)

BNP-Erhöhung > 3-fach gegenüber der alters-/

geschlechtskorrigierten Norm ${ }^{a}$

pulmonale Hypertonie (systolischer PAP

$>60 \mathrm{mmHg})^{\text {b }}$

a bei wiederholter Messung und ohne Erklärung durch andere Ursachen;

${ }^{\mathrm{b}}$ bei Bestätigung durch invasive Messung und ohne Erklärung durch andere Ursachen
LV-Dysfunktion: LV-EF $<50 \%$ ohne andere Ursache

Folge trotz klarer Indikation zum Klappenersatz (nach dem Auftreten von Symptomen) aber nicht operiert wurden und schließlich verstarben, gehen in die Analyse ein. Es bleibt unklar, warum diese Patientengruppe nicht operiert wurde bzw. ob es einen Einfluss durch Komorbiditäten gab, die eine Operation verhindert haben. Systematische Belastungstest wurden nicht durchgeführt. Zusammengefasst liegt keine Studie vor, die eine konsequente „Watchful Waiting“-Strategie mit einem frühzeitigen operativen Vorgehen vergleicht, laufende randomisierte Studien müssen abgewartet werden.

Das Risiko für einen plötzlichen Herztod bei asymptomatischer AS ist gering und beträgt etwa $1 \%$ pro Jahr. Dem stehen das Risiko eines chirurgischen Eingriffs und potenzielle Prothesen-Langzeitkomplikationen gegenüber (vor allem Endokarditis mit 50-fach erhöhtem Risiko gegenüber der Normalpopulation). Zusammenfassend muss bei Vorliegen von ungünstigen Prognoseparametern asymptomatischer Patienten ohne andere OP-Indikation ein niedriges Eingriffsrisiko vorliegen, um eine frühzeitige $\mathrm{OP}$ zu rechtfertigen. Bei den meisten Patienten ist aktuell eine Strategie der aktiven Nachverfolgung mit regelmäßigen Kontrollen möglich. Ob die Interventionsstrategien (TAVI vs. OP) einen Einfluss auf dieses Vorgehen haben, ist Gegenstand laufender Studien. Die aktuellen ESC/EACTS-Empfehlungen sind in > Tab. 1 zusammengefasst.

reduzierten Myokardfunktion einen Zusammenhang zur Symptomentwicklung und Prognose herstellen [9], sind aber noch nicht in den aktualisierten Leitlinien aufgeführt.

\section{Biomarker}

Die Plasmaspiegel von NT-pro-BNP bzw. BNP korrelieren mit dem Schweregrad der AS und einem früheren Symptombeginn. Darüber hinaus ist die BNP-Ratio bezogen auf alters- und geschlechtsspezifische Normwerte ein starker, unabhängiger Prädiktor des Überlebens für Patienten mit schwerer AS, was auch für asymptomatische Patienten mit normaler Ventrikelfunktion und das Überleben nach Klappenersatz gilt [10]. Normale BNP-Werte unterstützen auf alle Fälle das Zuwarten mit einer Intervention bei asymptomatischen Patienten. Bei einem Anstieg über das 3-Fache des alters- und geschlechtsbezogenen Normwerts sollte nach der neuen Leitlinie eine Intervention erwogen werden.

\section{Pulmonale Hypertonie}

Eine pulmonale Druckerhöhung aufgrund linksseitiger Vitien (postkapilläre pulmonale Hypertonie) lässt sich nur eindeutig im Rechtsherzkatheter differenzieren. Hohe Kleinkreislaufdrücke sind auch bei AS mit einer eingeschränkten Prognose assoziiert. Allerdings sind wirklich asymptomatische Patienten mit einem systolischen PAP > $60 \mathrm{mmHg}$ [8] selten.

\section{Prognosebesserung durch frühzeitige Operation}

Die Frage, ob bei asymptomatischen Patienten mit schwerer AS die Prognose durch frühzeitige Operation verbessert werden kann, bleibt kontrovers. Studien, in denen eine Übersterblichkeit in der konservativen Gruppe berichtet wurde (zusammengefasst in [6]) haben gravierende methodische Limitationen. Patienten, die ursprünglich als asymptomatisch eingestuft wurden, in der

\section{KURZGEFASST}

Die wirklich asymptomatische Aortenklappenstenose mit guter Ventrikelfunktion hat auch unbehandelt eine gute Prognose, es sind aber engmaschige Verlaufskontrollen notwendig, um den richtigen Operationszeitpunkt nicht zu verpassen bzw. das Risiko für den Patienten nicht zu erhöhen.

\section{Mitralinsuffizienz}

\section{Allgemeines}

Die Mitralinsuffizienz (MI) ist der häufigste isolierte Herzklappenfehler und nach der Aortenklappenstenose derjenige mit den häufigsten OP-Indikationen. Wichtig ist die Unterscheidung von primären Insuffizienzen mit strukturellen Veränderungen der Klappe und des Klappenapparates selbst und der sekundären Insuffizienz mit intakten Klappensegeln/Sehnenfäden und Deformierung durch veränderte Geometrie und Funktion von LV und/oder LA. Da eine asymptomatische schwere sekundäre MI eine Rarität darstellt und im Kontext der allgemeinen Herzinsuffizienzbehandlung steht, wird hier der Fokus auf die asymptomatische primäre Ml gelegt.

Laut Jahresbericht 2016 der DGTHG liegt die Rekonstruktionswahrscheinlichkeit bei allen isolierten Mitralklappeneingriffen nach wie vor nur bei $64 \%$. Bei Vorliegen einer primären MI sollte der Anteil deutlich höher (80-90\%) liegen. Die klappenerhaltende Rekonstruktion bei primärer MI ist sowohl mit Blick auf das Eingriffsrisiko als auch hinsichtlich der Langzeitergebnisse dem Klappenersatz überlegen (nicht jedoch bei sekundärer MI), wenngleich keine randomisierte Studie zum direkten Vergleich vorliegt. 
- Tab. 2 Behandlungsindikation der asymptomatischen, schweren primären Mitralinsuffizienz (Daten aus [8]).

LV-Dysfunktion/Vergrößerung (LV-EF $\leq 60 \%$ und/ oder LV-ESD $\geq 45 \mathrm{~mm})^{\mathrm{a}}$ B

Auftreten von Vorhofflimmern ${ }^{\mathrm{b}}$

pulmonale Hypertonie (systolischer PAP in Ruhe $>50 \mathrm{mmHg})^{c}$

LV-Vergrößerung (LV-ESD 40-44 mma), erfolgreiche MK-Rekonstruktion mit dauerhaftem Ergebnis wahrscheinlich, niedriges Operationsrisiko und Durchführung in einem Herzklappenzentrum

- bei Vorliegen eines Flail-Leaflets

- bei LA-Dilatation und Sinusrhythmus (Volumenindex $\geq 60 \mathrm{ml} / \mathrm{m}^{2}$ )

a Die Grenzwerte beziehen sich auf Erwachsene mit durchschnittlicher Statur und müssen bei ungewöhnlich kleinen bzw. großen Patienten adaptiert werden; ${ }^{\mathrm{b}}$ mit Bezug zum Vitium auch bei normaler LV-Funktion oder endsystolischer Ventrikelgröße (LV-EF > 60\% und LV-ESD $<45 \mathrm{~mm}$ ); ' wenn der Pulmonalisdruck die einzige Indikation darstellt, sollte er invasiv bestätigt werden vor einer Operationsentscheidung

\section{Natürlicher Verlauf und Prognoseparameter}

Asymptomatische Flail-Leaflet-Patienten mit normaler LV-Funktion und Sinusrhythmus haben ein geringes Risiko für einen plötzlichen Herztod (0,8\% pro Jahr [11]), bei stark symptomatischen Patienten steigt dieses Risiko jedoch erheblich an (12\% pro Jahr), zudem besteht eine Altersabhängigkeit mit ungünstigerer Prognose bei älteren Patienten. Bei einer jährlichen Eventrate von 10$12 \%$ (Entwicklung von Herzinsuffizienz, Vorhofflimmern) verbleiben nach 10 Jahren nur noch $10 \%$ ohne Operation oder Herztod.

\section{Prognostische Parameter}

Asymptomatischen Patienten mit schwerer primärer Mitralinsuffizienz wird eine Operation empfohlen, wenn eine Schädigung des linken Ventrikels erkennbar wird (LV-EF $\leq 60 \%$ oder endsystolischer LV-Diameter $\geq 45 \mathrm{~mm}$ ). Für Patienten mit Flail-Leaflet ist der Cut-off geringer (endsystolischer LV-Diameter $\geq 40 \mathrm{~mm}$ ). Auch eine deutliche Dilatation des linken Vorhofs mit erhaltenem Sinusrhythmus ist mit einer ungünstigeren Prognose vergesellschaftet. Darüber hinaus sind neu aufgetretenes Vorhofflimmern sowie eine pulmonale Hypertonie mit systolischem PA-Druck $\geq 50 \mathrm{mmHg}$ als ungünstige Prädiktoren für den postoperativen Verlauf identifiziert worden (siehe $>$ Tab.2). Als Hilfestellung kann auch bei diesem Krankheitsbild die Myokardfunktion, ermittelt durch den echokardiografisch quantifizierbaren globalen longitudinalen Strain (GLS), herangezogen werden, auch wenn dies aus methodischen Gründen noch nicht Eingang in die Leitlinien finden konnte. Eine Reduktion des GLS unter -21,7 war bei asymptomatischen Patienten und normaler EF mit einer höheren postoperativen Mortalität assoziiert [12].

\section{Belastungstests}

Auch bei der MI können Belastungstests bei primär asymptomatischen Patienten reduzierte Leistungsreserven demaskieren, was ebenfalls Einfluss auf die Prognose hat [12]. Ein echokardiografisch messbarer Anstieg des systolischen PA-Drucks unter Belastung auf $\geq 60 \mathrm{mmHg}$ wurde ebenfalls als prognostisch relevant berichtet [13]. Da aus bisherigen Daten nicht zu erheben ist, bis zu welchem Belastungslevel dieser Wert nicht erreicht werden sollte, und unsicher bleibt, wieviel zusätzliche prognostische Aussagekraft zum Ruhewert des Pulmonalisdrucks gegeben ist, wurde dies in den aktuellen Leitlinien nicht als Kriterium für eine Intervention aufgenommen.

\section{Biomarker}

BNP und NT-pro-BNP korrelieren gut mit der Symptomatik bei MI bzw. sind prädiktiv für die Entwicklung von Symptomen oder einer Ventrikelfunktionsverschlechterung, was als Klasse-I-Indikation für eine Operation gilt. Während der negative prädiktive Wert eines normalen BNP-Werts für Symptomentwicklung sehr hoch ist, was die Beurteilung einer tatsächlichen Beschwerdefreiheit erleichtern und eine „Watchful Waiting“-Strategie unterstützen kann, bleibt die Vorhersage einer ungünstigen Prognose, die eine Operation auf Basis von BNP-Werten rechtfertigt, schwierig. Obwohl eine Korrelation zwischen postoperativer Mortalität und Rehospitalisationsrate mit den Konzentrationen der natriuretischen Peptide gezeigt werden konnte, reichen die Daten für klare Behandlungsempfehlungen noch nicht aus [14]. Hilfreich kann die Bestimmung dennoch sein - vor allem als individueller Verlaufsparameter - um eine zunehmende Dynamik der Erkrankung festzustellen, welche das Erreichen definitiver Behandlungskriterien kurzfristig wahrscheinlicher macht.

\section{KURZGEFASST}

Patienten mit schwerer, asymptomatischer Mitralinsuffizienz haben eine gute Prognose, sofern die Ventrikelfunktion und -größe normal sind und keine pulmonale Hypertonie oder Vorhofflimmern vorliegen [15]. Belastungstests und Biomarker können hilfreich sein, bereits bestehende Leistungseinbußen zu objektivieren.

\section{Fazit}

Patienten mit nicht operierten, aber schweren asymptomatischen Klappenfehlern bedürfen einer besonderen Aufmerksamkeit in den Kontrolluntersuchungen. Neben klinischen Angaben tragen echokardiografische Verlaufsparameter, Belastungsuntersuchungen und ggf. weiterführende Diagnostik (Herzkatheter, Laborparameter) zur Festlegung des optimalen OP-Zeitpunktes bei. Die frühzeitige Erkennung ungünstiger Prädiktoren (u. a. Veränderung von Ventrikelfunktion und -größe) ist Voraussetzung für eine günstige Prognose der Betroffenen. 


\begin{tabular}{ll} 
ABKÜRZUNGEN \\
AS & Aortenklappenstenose \\
BNP & Brain natriuretic Peptide \\
EF & Auswurffraktion \\
ESD & endsystolischer Durchmesser \\
GLS & globaler longitudinaler Strain \\
LA & linkes Atrium \\
LV & linker Ventrikel \\
MI & Mitralinsuffizienz \\
MK & Mitralklappe \\
PAP & Pulmonalarteriendruck \\
TI & Trikuspidalinsuffizienz \\
V & Spitzengeschwindigkeit über die Aortenklappe \\
& \\
\hline
\end{tabular}

Der Autor gibt an, dass kein Interessenkonflikt besteht.

Literatur

[1] Otto CM, Burwash IG, Legget ME et al. Prospective study of asymptomatic valvular aortic stenosis. Clinical, echocardiographic, and exercise predictors of outcome. Circulation 1997; 95: 2262-2270

[2] Olsen MH, Wachtell K, Bella JN et al. Aortic valve sclerosis relates to cardiovascular events in patients with hypertension (a LIFE substudy). Am J Cardiol 2005; 95: 132-136

[3] Pellikka PA, Sarano ME, Nishimura RA et al. Outcome of 622 adults with asymptomatic, hemodynamically significant aortic stenosis during prolonged follow-up. Circulation 2005; 111: 3290-3295

[4] Rosenhek R, Binder T, Porenta G et al. Predictors of outcome in severe, asymptomatic aortic stenosis. N Engl J Med 2000; 343: 611-617
[5] Clavel MA, Pibarot P, Messika-Zeitoun D et al. Impact of aortic valve calcification, as measured by MDCT, on survival in patients with aortic stenosis: results of an international registry study. J Am Coll Cardiol 2014; 64: 1202-1213

[6] Généreux P, Stone GW, O'Gara PT et al. Natural history, diagnostic approaches, and therapeutic strategies for patients with asymptomatic severe aortic stenosis. J Am Coll Cardiol 2016; 67: 2263-2288

[7] Rafique AM, Biner S, Ray I et al. Meta-analysis of prognostic value of stress testing in patients with asymptomatic severe aortic stenosis. Am J Cardiol 2009; 104: 972-977

[8] Baumgartner H, Falk V, Bax JJ et al.; ESC Scientific Document Group. 2017 ESC/EACTS Guidelines for the management of valvular heart disease. Eur Heart J 2017; 38: 2739-2791

[9] Lafitte S, Perlant M, Reant P et al. Impact of impaired myocardial deformations on exercise tolerance and prognosis in patients with asymptomatic aortic stenosis. Eur ] Echocardiogr 2009; 10: 414-419

[10] Clavel MA, Malouf J, Michelena HI et al. B-type natriuretic peptide clinical activation in aortic stenosis: impact on long-term survival. J Am Coll Cardiol 2014; 63: 2016-2025

[11] Enriquez-Sarano M, Akins CW, Vahanian A. Mitral regurgitation. Lancet 2009; 373: 1382-1394

[12] Mentias A, Naji P, Gillinov AM et al. Strain echocardiography and functional capacity in asymptomatic primary mitral regurgitation with preserved ejection fraction. J Am Coll Cardiol 2016; 68: 1974-1986

[13] Magne J, Donal E, Mahjoub $\mathrm{H}$ et al. Impact of exercise pulmonary hypertension on postoperative outcome in primary mitral regurgitation. Heart 2015; 101: 391-396

[14] Johl MM, Malhotra P, Kehl DW et al. Natriuretic peptides in the evaluation and management of degenerative mitral regurgitation: a systematic review. Heart 2017; 103: 738-744

[15] Rosenhek R, Rader F, Klaar $U$ et al. Outcome of watchful waiting in asymptomatic severe mitral regurgitation. Circulation 2006; 113: 2238-2244 